\title{
Single Array and Response Modeling of Robust Design Experiments
}

\author{
Zaharah Wahid and Ming T. Tham
}

\begin{abstract}
Robu 1 st design experiments with single array were applied for the first time to optimization of rubber examination glove production in Malaysia. Seven controllable factors and one noise factor were combined in a single experimental setup at fixed levels also called a combined array or a single array. Two levels saturated fractional factorial designed was employed to elucidate the controllable factors that significantly affect the examination glove. The inclusion of the noise factor (relative humidity) allows a systematic study of the effect of process variation on product and process quality that can be expected under processing condition. The modeling was performed on the mean and standard deviation separately for the quality characteristics of interest (pinholes). The standard deviation model is used to investigate the possibility of reducing the process variations induced by uncontrolled factors while keeping the mean on target. In this paper, we demonstrate how response modeling derived from robust design experiments were employed to identify setting of the controllable factors so that quality characteristics are least sensitive to variation of noise factor.
\end{abstract}

Index Terms - Design of experiments, robust design, noise factor, multiple regression.

\section{INTRODUCTION}

Scientific experimentation in industrial quality improvement has recently received much attention in Malaysia. The use of robust design experiments has proven to be a powerful and cost effective strategy for building quality into the design of products and processes. This would be necessary to ensure longer term survival of the industry, especially in the event of stiff competition globally. Hence manufacturers would have to employ more specialized techniques to make further gains. In our case study seven controllable factors and one noise factor were combined in a single experimental setup, also called a combined array or a single array. The results and analysis of multiple regression models derived from the robust designed experiments including the noise factor are discussed. The modeling was performed on the mean and standard deviation separately for the quality characteristics of interest. The standard deviation model is used to investigate the possibility of reducing the process variations induced by uncontrolled factors not included in the experiments as noise factors (residual variation). In this paper, we demonstrate how response

Manuscript received May 11, 2012, reviewed June 13, 2012.

Z. Wahid is with the Department of Science in Engineering, Faculty of Engineering, International Islamic University Malaysia (e-mail: zaharahwahid@iium.edu.my).

M. T. Tham is with Department of Chemical and Process Engineering, Faculty of Engineering, University of Newcastle. modeling derived from robust design experiments were employed to identify setting of the design (controllable) factors so that quality characteristics are least sensitive to variation of noise factor. The knowledge gathered from the fitted models to the mean and standard deviation of the quality characteristics of concerned is very useful for economical and critical decision making.

\section{STRATEGY AND PlanNING}

\section{A. Robust Designed Experiments}

The strategy of robust process design is first, settings of controllable factors (process parameters), which produced desired quality characteristics with minimum variation. Then, a means of adjusting the average as necessary is developed. Robust design relies heavily on experimentation to assess the effects of different factors on product and process performance. Steinberg and Bursztyn [1] reported that robust design experiments are effective when it is possible to incorporate some variation directly in the experiments by mean of noise factors. When a noise factor is introduced in a robust experiment, variation is built directly into the results which enable more efficient modelling of the process variation. Seven controllable factors and one noise factor, two-level saturated fractional factorial 28-4 or L16 array were used, and their factor levels were fixed. Curing temperature profile, latex temperature, formers' temperature, percentage of calcium nitrate, percentage of calcium carbonate, oven temperature after coagulation dip and latex $\mathrm{pH}$ were identified as important controllable factors. The noise factor and the controllable factors were combined in a single factorial design array. In our case study, the noise factor that is the relative humidity was purposely included in the experiment in order to determine the controllable factor levels that are least sensitive to noise using average response. By reducing process variability we can better control process and can reduce the cost associated with development, manufacture and use. In this study, replication for each combination of each levels was made twice, so that not only process variation is included but also the variation due to uncontrollable sources which vary over longer time periods as well as variation introduced by resetting the factors. Also the effect of factors on variability as well as on average value could be assessed. The experimental design employed above has the property of being orthogonal.

A resolution IV of fractional factorial was also considered in the experimental plan so that all main effects are clear of two-factor interactions. The order in which these 16 trials runs were performed was randomized completely so as to reduce the effects of factors which were not controlled in the experimentation. 


\section{B. Model Building Based on Experimental Results}

All Two model strategies (location and dispersion modelling) and response modelling were employed. In the investigation, the pinhole was regressed. We want to choose a subset of explanatory variables that represent the phenomena under study. Separate analyses of the averages and the standard deviations were performed. The standard deviation model is used to investigate the possibility of reducing the process variations induced by uncontrollable factors not included in the experiment as noise factor (residual variation). A model containing all the main effects and two-factor interactions was fitted to the data. The forward selection, backward elimination and stepwise techniques were used to select a subset of explanatory variables. The variables were selected based on the significance of the t-ratio and by considering the $R 2$ and $R 2$ (adjusted) values that resulted from the inclusion of those variables. Another criterion for assessing the best model is the residual mean square also termed as error mean square MSE or the estimated standard deviation $\sqrt{\text { MSE. The smaller }}$ its value, the better the model fit.

The forward selection regression procedure builds a model by adding one effect (factor) at a time as long as the reduction in residual sum of square is substantial. The backward elimination regression procedure includes all the explanatory variables initially, but eliminates the variables one at a time starting with those having the smallest t-ratios. The step wise procedure uses either forward or backward selection to control the entry of variables into the model. This procedure is helpful in model building when there is large number of possible independent factors and the analyst is unsure which to include. Fitting the noise factor in a model with control factors has the advantage of providing a better understanding of the process.

\section{Modelling Location and Dispersion Effects on Pinholes}

The statistical software package Minitab was used to carry out the regression modelling [2].

Pinholes counts often follow a Poisson distribution. This is true in our case. This implies that groups with higher averages counts also have higher variability, because the mean and the variance in Poisson distribution are the same. This violates two of our assumptions: normality and constant variance. It can be shown that the square root of Poisson random variables does stabilise the variance and also provide a variable that has an approximate Normal distribution. A multiple regression was performed on the transformed data, that is $Y=\sqrt{ }$ No. of pinholes per 100 .

\section{RESUlTS AND DisCUSSION}

Analysis of variance (ANOVA) of the linear multiple regression fit is presented in Table II. The overall regression model obtained for pinholes is statistically significant with p-value (0.002). The estimated standard deviation of the errors about the regression line is 0.0625 with $R 2$ and $R 2$ (adjusted) $70.3 \%$ and $55.5 \%$ respectively. This implies that the model could probably reduce the variability in the pinholes by $55.5 \%$ respectively. With a model for pinholes now developed, we need to check for any inadequacies via a study of the residuals. The plot of the standardised residuals against fitted value indicates that the residuals are centred about the dashed line as given in Fig. 2 and there does not appear to be pattern. Fig. 1 shows the normal scores plot of the residuals of the model fitted. It seems to have a quite a good linear fit except for the two end points. On the whole, these plots show no obvious structural pattern and thus we may conclude that our model has no apparent inadequacies. These plots show that the underlying model assumptions are valid and that any conclusions drawn are appropriate.

The best equation for the mean square root pinholes was: $Y$ $=0.172+0.034 A+0.0374 B-0.0191 D-0.0524 G+0.0237 A B+$ $0.0167 A E+0.0315 B E-0.0220 B F-0.0160 C D$.

Table I shows that the important variables that affect the change in pinholes are factors $G$ (oven temperature before latex dip, $B$ (temperature of latex in dip tank) and $A$ (Curing temperature profile). These variables have high t-ratio -4.29 , 3.13 and 2.51 respectively. The negative sign of the coefficient for factor $\mathrm{G}$ implies that the pinholes are reduced when $G$ is set high. This equation also implies that lowering the curing oven profile and latex temperature will tend to give less pinhole. On the other hand increasing oven temperature after coagulation dip will also give less pinhole. The interaction between $D$ (\%calcium nitrate) and $F$ (calcium carbonate) was also found marginally significant $(p<0.063)$. Since none of the main factor effect for this interaction is significant, this suggests that the interaction is probably a random variation. Based on the inheritance principle when a two factor interaction is significant, at least one of the corresponding factor main effects is also significant [3]. It is also noted that there was an interaction between the noise and the controllable factor, that is $B E$ interaction as depicted in Fig. 3. The discovery of this interaction is very important information because we can understand the process better. Examination of Table III showed that interaction at $B 1 E 2$ yields less pinholes. This implies that factor $E$ has significant impact and contributes to variability in the process at some settings of the controllable factors. Thus, it is important to find setting of the controllable factors at which the effect of factor $\mathrm{E}$ is small. It appears that there is less percentage of pinholes when the latex temperature $(B)$ is set low and humidity $(E)$ is set high as given in Fig. 3. The goal here is to minimise the percentage of pinholes and minimise variability. However, humidity is a noise factor which cannot be controlled.

TABLE I: MULTIPLE REGRESSION RESULTS FOR PINHOLES.

\begin{tabular}{|l|c|c|c|c|}
\hline Predictor & Coefficient & $\begin{array}{l}\text { Standard } \\
\text { Deviation }\end{array}$ & $t$-ratio & $p$-value \\
\hline constant & 0.1716 & 0.0731 & 14.16 & 0.000 \\
\hline$A$ & 0.0304 & 0.0231 & 2.51 & 0.002 \\
\hline$B$ & 0.0374 & 0.0231 & 3.13 & 0.006 \\
\hline$D$ & -0.0191 & 0.0231 & -1.58 & 0.133 \\
\hline$G$ & -0.0524 & 0.0231 & -4.29 & 0.000 \\
\hline$A B(=D F)$ & 0.0237 & 0.0231 & 1.99 & 0.063 \\
\hline$A E$ & 0.0168 & 0.0231 & 1.39 & 0.180 \\
\hline$B E$ & 0.0315 & 0.0231 & 2.58 & 0.019 \\
\hline$B F$ & -0.0221 & 0.0231 & -1.82 & 0.086 \\
\hline
\end{tabular}


TABLE II: ANALYSIS OF VARIANCE FOR PINHOLES REgRESSION MODEL.

\begin{tabular}{|l|l|c|c|c|c|}
\hline $\begin{array}{l}\text { Source of } \\
\text { Variation.r. }\end{array}$ & $\begin{array}{l}\text { Degree } \\
\text { of } \\
\text { freedom. }\end{array}$ & $\begin{array}{l}\text { Sum of } \\
\text { Squares. }\end{array}$ & $\begin{array}{l}\text { Mean } \\
\text { Squares.r }\end{array}$ & F-value.r & p-value. \\
\hline Regression & 9.1 & 0.16659. & 0.01851 .1 & 4.74 .1 & 0.002 .1 \\
\hline Error.r & 18.1 & 0.07029. & 0.00391 .1 & .1 & .1 \\
\hline Total. 1 & $27 . .1$ & 0.23687. & .1 & .1 & .1 \\
\hline
\end{tabular}

TABLE III: MAIN EFFECTS FACTORS B AND G ON PINHOLES.

\begin{tabular}{|c|c|c|}
\hline Levels & \multicolumn{2}{|c|}{ Factors } \\
\hline & $\boldsymbol{B}$ & $\boldsymbol{G}$ \\
\hline $\begin{array}{c}\text { Average Response at High } \\
\text { Level (2) }\end{array}$ & 0.214 & 0.1301 \\
\hline $\begin{array}{c}\text { Average Response at Low } \\
\text { Level (1) }\end{array}$ & 0.1434 & 0.2273 \\
\hline Main Effect & 0.0706 & 0.0972 \\
\hline
\end{tabular}

TABLE IV: INTERACTION EFFECT OF BE ON PINHOLES.

\begin{tabular}{|c|c|c|}
\hline $\boldsymbol{B}_{1}$ (Low) & $\boldsymbol{B}_{2}$ (High) & \\
\hline 0.187 & 0.187 & $\boldsymbol{E 1}($ Low) \\
\hline 0.1 & 0.241 & $\boldsymbol{E 2}$ (High ) \\
\hline
\end{tabular}

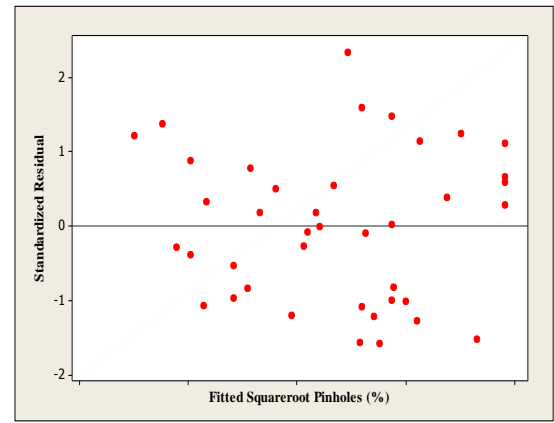

Fig. 1. Residuals versus fitted responses.

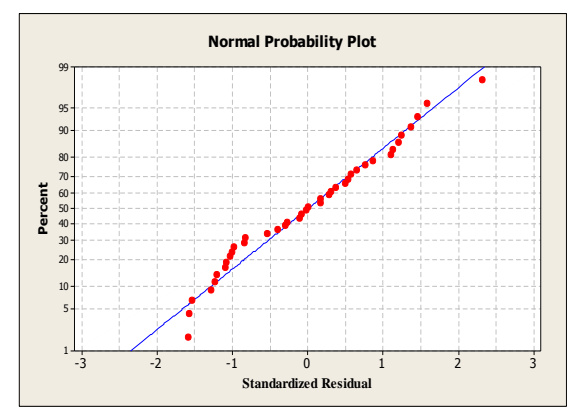

Fig. 2. Normal probability plot.

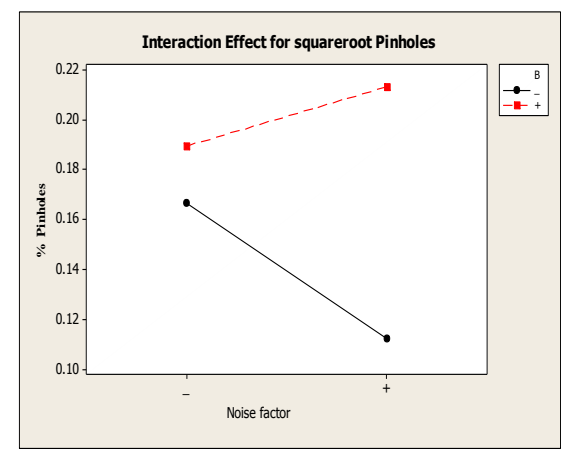

Fig. 3. Interaction effects for $\sqrt{ }$ pinholes.

We can see from Fig. 3 that when humidity is low, setting factor B either at low or high would yield similar percentage of pinholes. This indicates that factor B has almost no effect on average response when humidity is at low. But when humidity moves from low to high, we observed that $\mathrm{B}$ at low temperature percentage of pinholes drops from 0.187 to 0.099 . Thus, there is a decrease of pinholes by $47.05 \%$ but variation in the percentage of pinholes is increased. This is shown in Fig. 3 where $B 1$ has bigger slope as compared to $B 2$. On the other hand, if Factor $B 2$ is set at the high level, the percentage of holes increases by $28.87 \%$ but variability is small. There is conflict between level that minimize average pinholes and level that minimize variability. Since the percentage of pinholes is significantly reduced if we set at B1, it would be probably be best to set $B$ low. Thus by examining interaction effects we discovered that information about the $\mathrm{BE}$ interaction in this case is more useful than knowledge of the main effects on their own. Factor B affects both the average and standard deviation of the response. Even though it is important to reduce variability, the potential reduction in pinholes in the variability is small (28.9\%) compared to the potential reduction in the number of pinholes, which is greater $(47.10 \%)$. Thus we have to trade off variability in this case.

\section{CONCLUSION}

This study has attempted to improve the current performance of a rubber examination glove manufacturing process. The inclusion of noise factor in this study has provided crucial information and enhanced our understanding for choosing the appropriate process factors and their levels in rubber glove manufacturing. It shows that humidity could potentially influence the mean response of pinholes. The results of the study have shown that the variability in the responses could be minimised and there is significant potential for improvement.

This study also suggests that humidity influences pinholes. The discovery of interactions between controllable factors and humidity, suggest that the process can be made robust to changes in humidity by adjusting the latex temperature. The result of this finding is obviously very valuable and should be of commercial benefit to the company.

The results also suggest that some of the models developed from this study could potentially be used to facilitate changing manufacturing parameters to cope with changing customers' specifications

\section{ACKNOWLEDGEMENTS}

The authors acknowledge the Research Management Centre (RMC), International Islamic University Malaysia (IIUM) for supporting this research.

\section{REFERENCES}

[1] M. D, Steinberg and D. Bursztyn, "Dispersion effects in robust design experiments with noise," Journal of Technology, vol. 26, no.1, pp. 12-20, 1994.

[2] Minitab 2006 Minitab software, Release 15 and QC manual Inc. U.S.

[3] G. E. P. Box and Meyer, "Some new idea in the analysis of screening designs," Journal of research of the Nat. Bureau of standards, vol. 90, pp. 495-502, 1985 